\title{
An unusual complication of hip surgery: paradoxical coronary embolism of foreign material as a cause of acute MI
}

\author{
Michael Mallouppas ${ }^{1 *}$, BSc, MBBS, MRCP; Vassilios Vassiliou ${ }^{1}$, MBBS, MRCP, FHEA; \\ Martin Goddard ${ }^{2}$, MB BCh, FRCS, FRCPath; Bushra Rana ${ }^{1}$, MBBS, FRCP; \\ Denise Braganza ${ }^{1}, \mathrm{MBChB}, \mathrm{BSc}, \mathrm{FRCP}, \mathrm{PhD}$
}

\section{Department of Cardiology, Papworth Hospital, Cambridge, United Kingdom; 2. Department of Histopathology, Papworth Hospital, Cambridge, United Kingdom}

This paper also includes supplementary data published online at: http://www.pcronline.com/eurointervention/89th_issue/128

A 36-year-old man presented with crushing chest pain 10 days following an elective left hip replacement, which was complicated by a paraprosthetic fracture. Past medical history included psoriasis and left hip arthritis. He was a smoker but denied illicit drug use. The ECG showed inferior ST-segment elevation and anterior ST depression consistent with inferoposterior STEMI, and so he was transferred to our institution for a primary PCI. Emergency angiography revealed filling defects in the left circumflex (LCx) as well as the left anterior descending (LAD) (Figure 1A, Moving image 1, Moving image 2). Coronary thromboembolism was

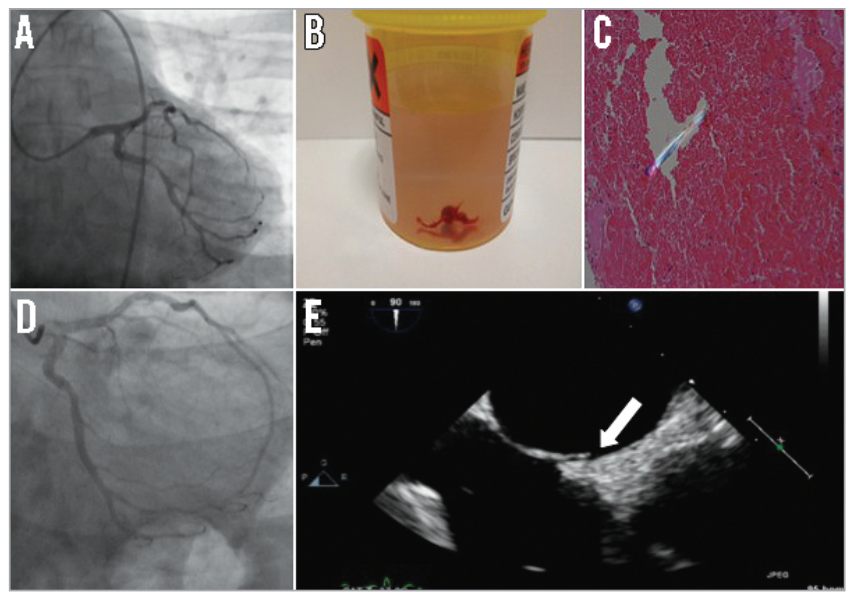

Figure 1. Angiography, histology and transoesophageal echocardiography images. A) Left circumflex view showing filling defect. B) LCx aspirate showing a yellow/white hard lesion covered with a red web-like thrombus. C) $x 200$ polarised histology of LCX aspirate showing platelet thrombus and a foreign plastic material, part of an intravenous peripheral plastic cannula. D) LCx view 48 hours later showing no thrombus and no associated plaque lesion. E) Transoesophageal echocardiogram confirming the presence of a PFO (arrow pointing to PFO tunnel). suspected and prolific thrombus aspiration was performed, following which his ECG normalised, the chest pain settled and TIMI 3 flow was restored. There were no identifiable lesions requiring intervention. The LCx aspirate was a yellow/white hard mass covered with thrombus. Histology revealed a platelet thrombus surrounding a foreign plastic material (Figure 1B, Figure 1C). Repeat angiography 48 hours later revealed smooth coronary arteries and did not show any lesions requiring intervention (Figure 1D, Moving image 3, Moving image 4). A transthoracic and transoesophageal echocardiogram with bubble study revealed a patent foramen ovale (PFO) (Figure 1E). CT pulmonary angiography and bilateral leg Doppler ultrasound excluded venous thromboembolism. On histology, the foreign plastic material was shown to be part of an intravenous peripheral cannula probably inserted during the hip operation acting as a nidus for thrombus formation following paradoxical coronary embolisation through the PFO. He subsequently underwent successful percutaneous PFO closure. Paradoxical coronary embolism is a rare but recognised cause of MI and must be considered in young patients presenting with acute MI, especially if more than one coronary is affected.

\section{Conflict of interest statement}

The authors have no conflicts of interest to declare.

\section{Supplementary data}

Moving image 1. Angiogram of left circumflex artery showing filling defect.

Moving image 2. Angiogram of left anterior descending artery showing filling defect.

Moving image 3. Repeat angiogram 48 hours later showing LCx with no thrombus and no obvious lesion requiring intervention.

Moving image 4. Repeat angiogram 48 hours later showing LAD with no thrombus or obvious lesion requiring intervention.

\footnotetext{
*Corresponding author: Department of Cardiology, Papworth Hospital, Cambridge, CB23 3RE, United Kingdom. E-mail:m_mallouppas@hotmail.com
} 